\title{
IXX. \\ Zur Lehre des Xenophanes. \\ Von
}

J. Freudenthal in Breslau.

Die in meiner Abhandlung Ueber die Theologie des Xenophanes entwickelte Ansicht, der Begründer der eleatischen Schule habe den reinen Monotheismus, den man ihm allgemein zugeschrieben hat, nicht gelehrt, ist Gegenstand verschiedener Beurteilung geworden. Hervorragende Gelehrte haben ihre Zustimmung ausgesprochen; von Anderen ist dagegen die alte, seit etwa zweitausend Jahren geltende Anschauung mit neuen Gründen verteidigt worden. Es sind Forscher vom Range eines Zeller und Diels ${ }^{1}$ ), die zwar einzelnen Ergebnissen meiner Schrift beigepflichtet, den Grundgedanken derselben aber als irrig bezeichnet haben. Bei dem hohen Werthe, der auf das übereinstimmende Urtheil dieser Männer zu legen ist, hielt ich mich für verpflichtet, ihre Grïnde der sorgfältigsten Prüfung zu unterziehen, um je nach dem Ausfall derselben entweder meine Ansicht offen zurückzunehmen oder sie nach Kräften zu verteidigen. Das Ergebnis dieser Prüfung möchte ich den Lesern dieser Zeitschrift vorlegen.

Nicht ein einzelner vieldeutiger Ausspruch des alten Philosophen oder späterer Berichterstatter war es, der gegen die Reinheit des xenophaneischen Monotheismus Zeugnis ablegen sollte; es war vielmehr eine Kette von Beweisen, die schon vor vielen

1) Zeller Deutsche Literaturzeitung Jahrg. 1886 S. 1595f. und Diels in diesem Archiv I S.97f. Auf diese Artikel verweise ich im Nachfolgenden, wenn ich die Namen $Z$ eller und Diels óhne weitere Angale nenne. Meine Schrift über Xenophanes bezeichne ich ler Kürze wegen mit ThdX. 
Jahren die landläufige Anschauung ${ }^{2}$ ) mir erschüttert und in oft erneuter Prüfung als stichhaltig sich erwiesen hatten. Der allgemein angenommenen Ansicht widerspricht die Geschichte der Gottesidee bei den Griechen; sie hat keine Stïtze an den Fragmenten der xenophaneischen Dichtungen und den Berichten glaubwürdiger Schriftsteller; sie wird durch unzweideutige Worte des Xenophanes selbst widerlegt.

Der Gottesbegrifi, an sich transcendenter Art, bietet verstandesmässiger Erfassung unüberwindliche Schwierigkeiten dar. Weder religiöse Vertiefung noch philosophische Erwägungen haben das Dunkel aufzuhellen vermocht, das ihn verhüllt und menschlicher Erkenntuis alle Zeit verhüllen wird. So darf es uns nicht Wunder nehmen, dass auch Religion und Philosophie des Griechentums die Gottesidee von offenbaren Inconsequenzen und schroffen Widersprüchen nicht haben befreien können; dass zumal der Polytheismus rom Bewusstsein des griechischen Volkes nie überwunden worden ist, der doch unserer Ueberzeugung nach den Begriff der Gottheit geradezu aufhebt. Den Glaubein, der ein einziges unendliches Wesen an die Stelle der Göttervielheit setzt, haben die Griechen nie bekannt und nie verstanden; 'der Himmel des Judentums und des Christentums mutete sie an, wie eine erkältende Oede ${ }^{3}$ ).

2) Der freundlichen und in allen wesentlichen Punkten zustimmenden Beurteilung meiner Abhandlung, die Lortzing in der Berliner Philol. Wochenschrift Jahrg. 1886 S. 1269 f. veröffentlicht hat, verdanke ich den Nachweis, dass bereits Bergk in der Griech. Litteraturgesch. II S. 419 Anm. 25 an dem reinen Morotheismus des Xenophanes gezweifelt, aber freilich eine weitere Begründung dieses $Z$ wreifels nicht gegeben hat. Bemerkenswert ist die das. ausgesprochene Vermutung, dass die Schrift De Melisso gar nicht von Xenophanes, sondern vou einem jüngeren uns unbekannten eleatischen Philosophen Jiandle.

3) Citat Friedländers in seinen lehrreichen Erörterungen über Griechische Mythologie (D. Rundschau XIV S. 88f.). Niemand hat die Bedeutung des Polytheismus für die Entwickelung des griechischen Denkens cindringlicher entwickelt, als Lehrs, der feinsinnige Kenner altgriechischer Religion und Litteratur. Yehrere tief eindringende Untersuchungen, in denen er den falschen Ansichten und Folgerungen über den Monotheismus der Griechen entgegentritt, bestätigen die obein dargelegte Anschauung; insbesondere sei auf die Abhandlung über 'Gott, Gïtter und Jämonen', sowie auf den gegen Preller gerichteten Aufsatz üher 'Naturreligion' (Popul. Aufs. 2140f. 261 f.) hingewiesen. 
In der Blütezeit griechischer Kunst und Philosophie haben die edelsten Dichter, dic tiefsten Denker den Grundgedanken des Polytheismus festgchalten: das lehren uns Pindar, Aeschylos und Sophokles ebensowie Sokrates, Platon und Aristoteles. Aber auch in der ' Zeit des Niederganges, als fremde Einflüsse die eigenartigen Anschauungen des griechischen Volkes in wesentlichen Zügen umgestaltet hatten, bliel, der Glaube an die Vielheit der Götter in wenig geschwächter Kraft bestehen. Die überlieferten religiösen Vorstellungen wurden veredelt, die Götterwelt einer höchsten Einheit untergeordnet; aber die Vielheit der Göttergestalten ward nie ganz aufgehoben, der Monotheismus dem Atheismus gleichgestellt ${ }^{4}$ ).

Es wäre denkbar, widersprïiche aber aufs entschiedenste dem Gange der geschichtlichen Entwickelung, wenn einer jener alten griechischen Philosophen, die noch mitten in verschwommenem Hylozoismus stehen ${ }^{5}$, wenn der Mann, über dessen bäurisches Denken Aristoteles geringschätzig aburteilt, einen Gedanken ausgesprochen hätte, dem Sokrates, Platon und Aristoteles nahe gekommen sind, den sie aber niemals in voller Klarheit und Entschiedenheit erfasst haben. Die Schwierigkeit einer solchen Annahme ist Diels nicht entgangen, und er selbst macht daher meiner; von ihm bekïmpften Anschauung das schwer wiegende Zugeständnis (S. 98): 'Die ganze Entwicklung der Gottesidee bei den Griechen drüngt zu der Auftassung hin, wie sie hier .... entwickelt ist.'

Hat nun aber Xenophanes den bedeutsamen Schritt in Wirklichkeit gethan; hat er mit der Grundlehre seiner Religion und dem Bewusstsein seiner Zeitgenossen so entschieden gebrochen, wie man lange Zeit angenommen hat; hat er sich mit Ausschluss aller Vielgötterei zu einer rein monotheistischen Lehre bekannt, dann

4) Ausser den ThdX. S. 11 f. gegebenen Belegen vgl. Philodemos De piet. p. 84 G.; Cicero De nat. deor. I 13, 32 ; Lucian De morte Peregr. c. 21 ; Dio Cass. (Xiphil.) 67, 14. Eine reichhaltige Sammlung von Belegstellen aus späteren Schriftstellern giebt Illgen Denkschr. d. histor.-theol. Ges. II S. 127f. und Semisch Justin d. Märtyrer II S. 100f. Sehr lehrreich sind auch Gibbons Bemerkungen in Hist. of the decline etc. II ch. XVI.

5) ThdX. S. 24. Erst mit Anaxagoras schwindet der alte hylozoistische Irrtum, freilich um bei Diogenes von Apollonia und den Stoikern in anspruchsvoller philosophischer Begründung wiedcrzukehren. 
dürfte man erwarten, dass die Geschichte hiervon in unzweideutiger Weise Kunde gebe. Denn kein Gedanke konnte doch seine Zeitgenossen so tief erregen, nichts konnte denen, die über seine Philosophie berichteten, der Erwähnung würdiger erscheinen, als jene unerhörte Lehre. Aber ein, solches unumwundenes Zeugnis für Xonophanes' Monotheismus tinden wir nirgends. Es giebt kein einziges Bruchstück seiner Dichtungen, keine einzige. Mitteilung zuverlässiger Berichterstatter, die hierfür angesehen werden könnte. Weder Platon noch Aristoteles, weder 'Theophrast noch einer der späteren Darsteller philosophischer Lehrmeinungen teilen über Xenophanes irgend etwas mit, was im Sinne eines entschiedenen Monotheismus verstanden werden dürfte, wie schon früher nachgewiesen worden ist (ThdX. S. 20 f.) und hier noch näher erörtert werden soll. Alle Kunde von dem angeblichen Monotheismus des Xenophanes stammt lediglich aus der fälschlich dem Aristoteles beigelegten unglaubwürdigen Schrift De Melisso Xenophane Georgia. Wer möchte ihr mehr vertrauen, als den echten Fragmenten der xemophaneisehen Jichtungen und den Aussagen zuverlïssiger Berichterstatter $\left.{ }^{\circ}\right)$ ?

Denn auch dieses muss behauptet werden. Es findet sich in guten Quellen nicht allein kein sicheres Zeugnis für die strittige Lehre des Jenophanes, sondern es wird die ihr entgegengesetzte Yorstellung von einer Göttervielheit in den Fragmenten des Philosophen und in den Berichten alter Geschichtschreiber so klar ausgesprochen, dass man zu den gezwungensten Deutungen seine $\mathrm{Zu}$ llucht nehmen musste, um die Angaben der pseudo-aristotelischen Schrift retten zu können. In Fr. 1 lehrt Xenophanes:

6) Gelehrte wie Zeller und Diels konnten diese Schrift, deren Unzuverlässigkeit sie selbst dargethan haben, als eine für Erkenutniss der xenophaneischen Lehre nutzhare Quelle nicht länger ansehen und haben sie in der That in ihren Besprechungen meiner Abhandlung kaun noch genannt. Wenn dagegen ein griechischer Sprache und Litteratur wenig kundiger Recensent dieser Abhundlung auch dem über Xenophanes handelnden Abschnitte der ps.-aristotelischen Schrift wieder hohen $W^{\prime}$ erth zuerkennt, so bedarf das keiner Widerlegung, zumal nachdem durch Apelt erwiesen ist, dass ihren Verfasser die eigenen Worte des Xenophanes gar nicht inehr vorgelegen hithen (Jahrb. f. class. Phil. 1886 \$. 759). 
'Fin (iott ist unter fïittern und Menschen rler Grïsste'; in lir. 21 mahnt or, 'don Göttern inmer rechte Verehrung zu erwoisen'; in Fr. 14 crklaït er, 'dass Niemand in Klarheit kundig sei dessen, Wals er, ler Dichter, über die Götter und das Weltall lohre'7). - Zu don \%engnissen der Fragmente treten die Aussagen alter Borichterstatter ergänend hin\%. Nach Aristoteles hat sich Ienophanes gegen die Moinung, dio Gïtter seien entstanden oder vergiinglich, ausgesprochen, weil dio (onsequen\% derselben sei, dass es \%u irgonl oiner Zeit keine Götter gebe (ThdX. S. 10). Nach 'Theophrast hat or erkliirt, dass keine Gewaltherschaft unter den (Göttern bestohe und dass sio bediifnislos ") seien : ihre Existenz wird also vorausgesetzt. Nach Poseidonios bei Cicero (De divin. I 3, 5) ist Xenophanes der einzige Philosoph, der die Existenz der Göttei annimmt, dio IVeissigung aber verwirft.

So oft wird unbefangen einer Mehrheit göttlicher Wesen von lem Manne gedacht, der dom Glauben und den Sitten seines Volkes mit der grössten Schärfe gegenüberzutreten gowohnt war, der gegen die religiöse I'eberlieforung mit einem Ingrimme kïmpft, dem in

i) In dor wichtigen Abhandlung, dio Gomperz über Heraklit in den Sitzungsberichten der Wioner Akatl. d. Wissensch. $1886 \mathrm{~S} .997 \mathrm{f}$. veröffentlicht. hat, berichtigt er meine Uebersetzung von $\pi x^{2} \tau \omega \nu$ in Fr. 14, das hier wohl nicht. 'Alles' sondern 'das All' betloutet: $\tilde{\sigma} \sigma \alpha \alpha \hat{\varepsilon} \gamma(\omega$ aber auf $\pi \dot{v} \tau \omega v$ zu be\%ichen und 'was ich das All nenne' zu verstehen, da\%u liegt kein genügender

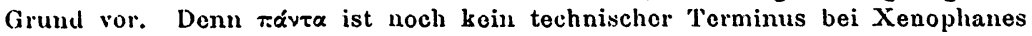
goworden, wie aus dem häufigen Gobrauche des Wortes in gewöhnlicher Be-

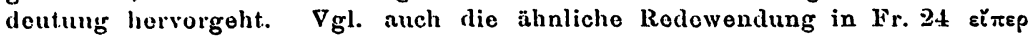

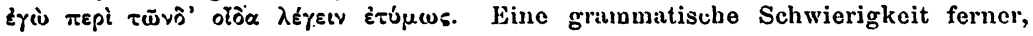
die Diels (S. :S) in den Worten findet, vermag ich nicht anzuerkennen, da

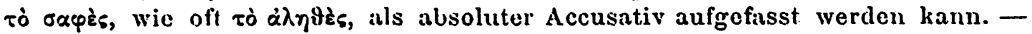
Von nicht goringer Bodoutung muss übrigens Allen, welche Gomperz als atusgezeichneten Kenner der griechisehen Philosophie hochschïtzen, seine Erklitrung sein, dass er das Hauptergebnis noiner Schrift 'unbedingt unterschreibe'.

9) Dem Wortlante dieses Borichtes zufolge muss er auch behauptet baben, dass die Gütter durchaus hören und sehen. Denn das Subjekt \%u áxójatv $\tau \varepsilon$ xai ¿pãv muss deı voraufgehenden $9 \varepsilon \bar{\omega} v$ entnommen, kann also nur sein, und Diels l)oxogr. 1. 704 übersetzt daher ganz richtig deos usquequaque audire ef videre. Doch liegt hier wohl nur eine ungenaue Unschreibung ron Fr. 2 ror. 
ahnlicher Weise ron griechischen Denkern wohl nur noch Heraklit Ausdruck gegeben hat. Einem solchen Manne ist eine Anbequemung an den Glanben seiner Zeitgevossen und ihre Sprechweise nicht zuzutrauen. und wenn ihm vielleicht ein oder das audere Mal ein Ausdruck entschlïpft sein mag, der den Tolksmeinungen besser entsprach als den eigenen Anschauungen. so kann er doch nicht so oft und so entschieden sich selbst verleugnet haben. Wie der gegnerischen Auschaumg zufolge angenommen werden müsste.

Das wichtigste ron den angeführten Bruchstụcken ist das erste:

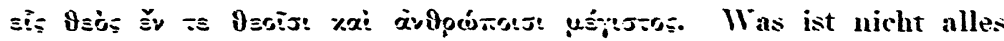
versucht worden ${ }^{3}$ ), um den offenbar polytheistischen Sinn des Bruchstückes mit der ühlichen Aufiassung der renophaveischen Theologie zu rereinigen? Es lehre zwar unverkennbar den Polytheismus, giebt Kern zu: aber es stamme, meint er, aus einer Zeit. in der Jenophanes noch nicht Monotheist war. - Das Fragment klinge allerdings polytheistisch; aber Senophanes, in Wahrheit Monotheist. habe eben nicht den Mut gehabt, der Volksmeinung offen gegenüberzutreten, so urteilt Brandis. - Dass hier ein Widerspruch zwischen den Worten des Philosophen und der ihm zugeschriebenen Anschauung ron der Gottheit vorliege, gesteht auch Farsten zu. Er glaubt aber; diese Schwierigkeit durch die Annahme heben zu können, die griechische Sprache habe Xenophanes die für den monotheistischen Gedanken passenden Worte nicht darzubieten vermocht. - Keiner ron diesen Meinungen pflichtet Dilthey bei. Ihm zufolge müssen wir den Widerspruch zwischen dem Sinn dieser Worte und der monotheistischen Lehre des Tenophanes ofien ancrkennen. haben ihn aber auf den tiefen Gegensatz zurückzuführen, der zwischen den Forderungen des Denkens und den Bedürfuissen des Gemütes schon für Xenophanes bestanden haben soll.

Dass alle diese Lösungsversuche unzureichend sind, hat schweigend oder in ausdrücklichen Worten Zeller anerkannt, und ich habe es des näheren zu begründen versucht (ThdX. S. 4 f.). Wertvoll aber sind sie uns durch das ihnen zu Grunde liegende Zugeständnis: dass in dem ersten Fragmente des Xenophanes das l)asein

:) S. Thix. S.4f. 
Bines höchsten Gottes gelehrt. eine Mehrheit von Göttern aher nicht \%uriickgewiesen werde.

Zeller vertritt eine andere Ansicht. Er hat schon in seiner

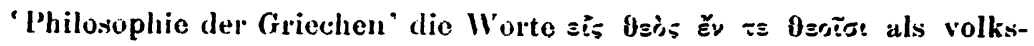
tiimliche Redeweise aufgefasst, welche den eigentlichen Gedanken les Xenophanes nichts angehe, und ebenso urteilt er in der $\mathrm{Be}$ sprechung meiner Schrift. Aber auch seine bewundernswerte Kunde griechischer Philosophie und jüdischer, wie christlicher Theologie hat ihn koin zweites Beispiel einer Thatsache kennen gelehrt, die seiner Erklärung zufolge hier anzunehmen wäre. Weder bieten die von Antisthenes erzählten Anekdoten, in denen von Göttern die Rede ist, noch die Schriften jüdischer und christlicher Dichter und Prosaiker, die von einem 'Gott der Götter' sprechen, ein solches Analogon dar. Denn Antisthenes' monotheistische Lehre ist lediglich aus Philodemos (De piet. p. 72 Gomp.) bekannt, den, oder dessen Quelle, Cicero (De nat. deor. I 13, 32) ungenau übersetzt hat.

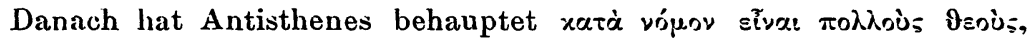

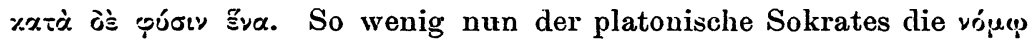
bestehende Gesetze, die Geschwister der ewigen Naturgesetze, für unverbindlich erklärt; so wenig Aristoteles das positive Gesetz, weil es nur vóụ besteht, verwirft, so wenig wird Antisthenes, weil er den Glauben an die Vielheit der Götter für einen konventionellen hält, von ihm sich vollständig losgesagt haben. Dass er das nicht gethan hat, beweist Tertullians Mitteilung (Ad nationes II 2), der zufolge Antisthenes auf die Frage, ob es Götter gebe, geantwortet haben soll: nescio nisi ut sint expedive. Dasselbe lehren die Erzählungen von Antisthenes' 'Theiluahme an religiösen Gebräuchen und von der Ehrfurcht, die sein treuer Schüler Diogenes den Heiligtümern der Götter bezeugte (D. L. VI 4. 37. 64). Antisthenes hat also in der Theorie zum Monotheismus sich bekannt, in ähnlicher Weise aber wie die Skeptiker, wie zahlreiche römische Denker, wie in neuerer Zeit Hobbes dem Volksglauben sich anbequemt und im praktischen Leben die Staatsgötter ebenso gelten lassen, wie die staatlichen Einrichtungen, deren Autorität er ebenfalls prinzipiell bestritten hatte (s. 'ThdX. S. 14). Dass er unter solchen Umständen, zumal im Verkehr mit anders Denkenden, den 
Gebrauch von 9 soi nicht zu meiden brauchte, ist einleuchtend ${ }^{10}$ ). Uebrigens spricht er von veoi entweder in ganz farblosen Formeln, wie bei Xenophon (Symp. 8,4) oder vom Standpunkte des Volkes aus, wie bei D. L. VI 1, oder der Wortlaut ist gänzlich unverbürgt, wie bei Joh. Dam. Par. sacr. II 13, 76. IV p. 199 Mein. und in ähnlichen Anekdoten, deren Fassung von der Willkür der Sammler

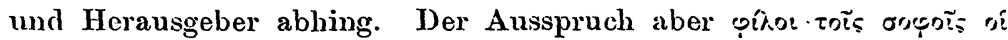
Orsi gehört dem Diogenes an (D. L. VI 37) ${ }^{11}$ ).

Wenn ferner Monotheisten der Bibel die Worte 'Gott der Götter' nachsprechen, so geschieht das, weil sie kein Bewusstsein von dem ursprünglich polytheistischen Sinn derselben haben. Der durch heilige Schriften gerechtfertigte Gebrauch einer ïberlieferten festen Formel kann daher nicht mit Redewendungen verglichen werden, in denen Xenophanes von den Göttern spricht. Er, der mühsam aus dem Polytheismus des Griechentums sich emporgerungen hat, dessen ungestümer Wahrheitsdraug ihn in den schroffsten Gegensatz zu Volksmeinungen und Volkssitten stellt, desser Freimut ihn die schärfsten Worte als Ausdruck seiner revolụtionären Gedanken wählen lässt, soll zum ersten Male die einfache, aber fundamentale Lehre ausgesprochen haben, class es nur Einen Gott gebe; er soll dic Vielheit göttlicher Wesen zurückgewiesen haben - das aber in Worten, die durchaus den von ihm so ingrimmig bekämpften Vorstellungen des Volkes entsprechen! Eine solche Annahme ist doch wohl nicht als wahrscheinlich anzusehen.

1') Lnbegründet erscheint demuach Bernays' Vermutung (Lucian und dic liyniker S. 94 A. 15), die bei D. L. VI 4 berichtete Erzählung "beziehe sich nur auf zufälige Anwesenheit bei einer einleitenden Ceremonic', 'der wirblichen Weihen sei Antisthenes nie gewürdigt worden'. - Diogenes' fromme Gesinnung will Julian (Or. VI l. 199 B) nicht bezweifeln; Krates Götterglauben bezeugt das von Julian (das. p. $199 \mathrm{D}$ und VII p. $213 \mathrm{~B}$ ) angeführte Bruchstück.

11) Ebensowenig ist der Alexandriner Philon mit Xemophanes zu vergleichen. Er, der Nonotheist, nennt allerdings die Gestirne sichtbare Götter (p. 6, 16; 14, 46; II 1. 214, 26;643 Mang.). Aber es ist bekannt genug, dass er sehr oft die widersprechendsteu Bestimmungen mit einander verknüjft, und lass er in seiner ungenauen, ja wirren Terminologie seinen Quellen oft AusIrücke entlehnt, die er keineswegs im eigentlichen Sinne angewendet wissen will: '(jïtter' sind ihm nur Wesen überirdischer Art, 
Auch Jiels scheint sie fïr unwahrscheinlich \%u halten. Denn er schlïgt einen andern Ausweg ein, auf den als einen möglichen schon Zeller (S. 1596) hingewiesen hatte, wohl den leteten, den es gicbt: er erkliint das erste Fragment für unecht. 'Fr. 1', so äussert er sich (das. S. 9S), 'steht bei Clemens in so gefährlicher Nachbarschaft mit sicher Aristobulischen Fälschungen, dasis hierauf, wie auf andere ebenfalls nicht unbedenkliche Xenophanescitate des Clemens nicht zu bauen ist'. Wie gern man sich wun auch in allen; die Quollen griechischer Philosophie betreffenden Fragen Diels" kundiger Führung überlassen möge, in dieser Ansicht wird man ihm nicht folgen dürfen.

Nicht sprachliche oder sachliche Gründe, sondern lediglich die böse Nachbarschaft der aristobulischen Fälschungen lässt Diels das bisher nie angefochtene erste Fragment als verdächtig erscheinen. Aber längst hat ja Valckenaer (De Aristobulo p. $106 \mathrm{f}$.) nachgewiesen, dass das in nicht unbeträchtlicher Entfernung von unserm Fragment stehende längere Citat aus Pseudo-Aristobul ${ }^{12}$ ) gar nicht in den Zusammenhang der clementinischen Schrift passt, dass es, wie viele ähnliche Einschiebungen ${ }^{13}$ ), notwendig gestrichen werden muss.

Doch Diels wird vielleicht die meiner Ueberzeugung nach wohlbegründete und auch von $W$. Dindorf in seiner Ausgabe des Clemens gebilligte Ansicht Valckenaers zurückweisen und den Text des Clemens für unversehrt erklären; aber der gegen das xenophaneische Fragment von ihm ausgesprochene Verdacht ist mit der Nähe der aristobulischen Citate doch nicht zu begründen. - Clemens ist vielleicht dei beleseuste unter den Vätern der griechischen Kirche. Er hat keineswegs, wie die Verfasser ̈̈hnlicher Schriften, seine Citate aus einigen -späten Sammelwerken zusammengestoppelt, sondern neben solchen auch eine grosse Zahl alter echter Original-

13) Die Gründe, die Lobeck, Grätz, Kueuen, Joël für die Unechtheit des dem Aristobul zugeschriebenen Commentars beigebracht haben, hat eine eingehende Untersuchung als durchaus zutreffende mir erwiesen und durch weitere Beweise bestätigt.

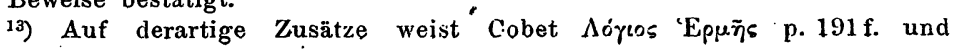
a. a. 0 . hin, 
schriften gelesen und benutzt. Von beklagenswerter Kritiklosigkeit und unglaublicher Flüchtigkeit hat er durch Trugschriften sich oft täuschen lassen; aber neben Excerpten aus offenbaren Fälschungen teilt er uns die erlesensten Ueberreste aus alter guter Zeit mit. Wer möchte diese um der oft sehr anrüchigen Nachbarschaft willen zurückweisen? - Clemens stellt altgriechische, jüdische und christJiche Schriftsteller, echte und untergeschobene Schriftwerke sorglos neben einander. Homer erscheint neben Jesaias und den Korintherbriefen, Platon neben Moses und dem Lukas-Evangelium, Kleanthes neben den Sprïchen Salomons, dem Siraciden und dem Briefe an die Thessaloniker. Hiob und Sophokles, Euripides und der Pastor des Hermas, Menander und die falsche Sibylla werden in einem Atem genannt. Und bei dieser Mischung aller Elemente dürften wir ein Citat wegen der Nühe eines pseudepigraphen Stïckes für unecht erklären? dürften ein Bruchstïck aus Xenophanes rerdächtigen, weil ein, noch dazu durch einen grossen Zwischenraum von ihm getrenntes Citat aus Ps.-Aristobul voraufgeht?

Das in unserm Falle zu thum, muss auch folgende Erwägung uns abhalten. Die Verse des Xenophanes sind von Citaten umgeben, die Clemens nicht blos in den Stromateis, sondern auch in der Cohortatio in gleicher Reihenfolge aufführt. Man vergleiche:

Cohortatio p. 59 Pot.

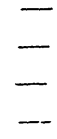

Antisthenes

Xenophon

Sibylla

Kleanthes
Stromateis p. 714 Pot.

Aristobul

Solon

Sapientia Salomonis

Platon

Antisthenes

Xenophon

Sibylla

Xenophanes

Bakchylides

Kleanthes

Die Citate aus Antisthenes, Yenophon, der Sibylla und Kleanthes stammen, wie diese Zusammenstellung lehrt, aus derselben Quelle und einer solchen, die nichts mit den ps.-aristobulischen Fälschungen gemein hat. Wer trotz dieses Umstandes das Fragment 
der Nenophanes um der entfernten Nachbarschaft des Aristobul willen verurteilt, der mïsste notwendig ach die der Fälschung nïher stehenden Aus\%äge aus anderen Schriften für unecht erklären: ihre Echtheit kann abor nicht angefochten werden ${ }^{*}$ ) und schüt t so auch die Verse des Xenophanes gegen jeden Verdacht.

Nur in wenigen Fälen wird man im Stande sein, nicht blos die gogen die Echtheit von Bruchstücken alter Dichtwerke vorgebrachten Vordachtsgründe zu entkräften, sondern auch positive Beweise fiir sie anzuführen. Auch an solchen fehlt es den angegriflenen Versen nicht. Mit dem ersten Fragment fallen nämlich, wie Diels selbst andeutet, auch die bei Clemens sich unmittelbar anschliessonden Bruchstüicke. Es sind die bekannten

Fr. 5

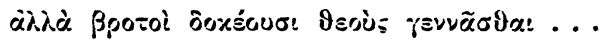

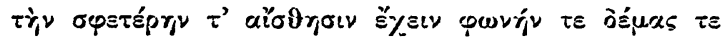

14) Das Citat aus Xenophons Memorabilien findet sich nicht in unseren Handschriften Xenophons, wohl aber bei Stobaeos (Anthol. II p. 15 Wachsm.) und hier noch um einen Satz vermehrt. Der Zusammenhang, in dem es er-" scheint, weist auf den Bearbeiter der Apomnemoneumata hin, den. uns besonders Dindorf und Schenkl kennen gelehrt haben. An eine Fälschung A ristobuls aber hätte Schenkl (Sitzungsber. der Wiener Akad. LXXX S. 128) nicht lenken sollen. Denn Niemand wird einen Grund angeben können, der einen Anbänger der biblischen Lehre zu dieser Umschreibung des voraufgehenden Satzes hätte veranlassen können, und eine Annäherung des Ausdrucks an biblischen Sprachgebrauch, von der Schenkl spricht, ist nicht zu rkennen. Ferner finden wir unter den zahlreichen Falsificaten Ps.-Aristobuls kein einem griechischen Prosaiker untergeschobenes Stück. Endlich würden wir ein gefälschtes Citat aus Xenophon schwerlich im Florilegium des Stobaeos antreffen, der vielntehr einen vortrefflichen Xenophontext benutzt haben muss, wie wir denn ihm die Rettung mehrerer anderer in den Handschriften fehlender Stücke der Apomnemoneumata und zahlreiche Verbesserungen der handschriftlichen Ueberlieferung verdanken (Schenkl das. S. $100 \mathrm{f}$.). - Cobet, der für Fälschungen und Entstellungen altgriechischer Schriften den schärfsten Blick hat, zweifelt nicht an der Echtheit des von Clemens und

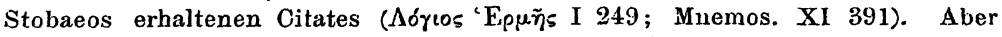
er irrt, wenn er als gemeinsame Quelle beider Musonios ansieht, was schon wegen des Citates aus der Sibylla nicht angeht. Auch ist nicht abzusehen, warum das ganze Stück nur in der Cohortatio am rechten Orte stehen und aus derselben in die Stromateis übertragen sein soll. Die oben gegebene Zusammenstellung widerlegt diese Vermutung. 
und Fr. 6

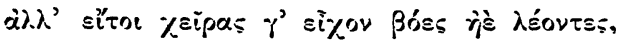

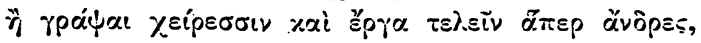

xai $x \varepsilon$ ปे

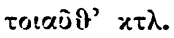

Wer aber möchte in diesen prïchtigen, das edle Pathos einer alten kräftigen Zeit atmenden Versen das Werk eines späten falschmünzenden Stümpers erblicken ${ }^{15}$ )? Wer möchte glauben, dass ein Mann vom Schlage eines Ps.-Aristobul Verse ron der Wucht dieser Fragmente gedichtet habe $\left.{ }^{16}\right)$ ?' Wer kamn Machwerke wie dic Ps.Orphika Aristobuls diesen Dichtungen auch nur nahe stellen? Und fermer. Sämtliche ps.-aristobulische Gedichte sind, wie keines $N$ achweises bedarf, verfasst worden, um das hohe Alter der biblischen Lehren und die Abhängigkeit des Heidentums vom Judentum zu erweisen und die Religion des Judentums oder Christentums gegen allerlei Angriffe zu schützen. Wann aber hat ein Jude oder Christ im Interesse der biblischen Lehre einem alten griechischen Dichter Verse von so entschieden polytheistischem Klange in den Mund gelegt, wie sie in Fr. 1 vorliegen? Wann ferner hat ein Bekenner der biblischen Religion zur Verherrlichung seines. Glaubens gegen die anthropomorphen Vorstellungen von der Gottheit geeifert, wie es in Fr. 5 und 6 geschieht? Gerade den Anhängern der Bibel wurde ja, wie besonders aus Celsus, Porphyrios und Julianus A postata zu ersehen ist, vorgeworfen, lass ihre heiligen Schriften Gott menschliche Gestalt beilegen. Darum halten Philon, Josephus, Ps.-Aristobul und zahlreiche Kirchenväter eine Verteidigung ihrer Religion gegen

15) Die Fr. 5 u. 6 sind bei Clemens nicht unversehrt erhalten. Abcr Niemand wird deshalb jhre Echtheit verdäcbtigen, der den bedauernswerten Zustand kennt, in welchem zahllose Bruchstücke philosophischer Schriften aus vorplatonischer Zeit uns jetzt vorliegen.

ig) len weiten Abstand der xenophaneischen Verse von Dichtungen, die in vermeintlichen Interesse des biblischen Jonotheismus alten griechischen Schriftstelleru untergeschoben sind, ersicht man am besten aus eiuer Vergleichung jener Bruchstūcke mit den ps.-pythagoreischen Versen bei Ps.-Justinus (De mon. c. 2 p. $105($ ) und den daselbst (p. 104f.) und von Clemens (str. p. 717 ff.) citirten Ps.-Aeschylos, Ps.-Sophokles, Ps.-Epicharm, Ps.-I)iphilos und ähnlichen Fälschungen. 
dorartige Vorwiirfe fïr notwenlig. Dass aber von jüdischen oder christlichen Apologeten Verse gefiilscht seien, um in leidenschaftlichen Worten den Glauben an menschenïhnliche Götter \%u bekämpfen, ist meines Wissens nicht nachzuweisen, während derartige Ausfälle von griechischen Philusophen gegen die Roligion ihres Volkes häufig sind. so gerït denn Diels, der un den Monotheismus des Xenophanes zu retten, das erste fragment Ps.-Aristobul zuschrcibt, in ein schwieriges Dilemma. Entweder ist der Sinn des Fragmentes ein polytheistischer: dann kann es nicht von Ps.-Aristobul und von keinem Bekenner einer monotheistischen Religion herrihren, oder es enthält die monotheistische Lehre: dann liegt keine Veranlassung vor; es als unecht anzusehen.

Wenn nun zum Schlusse. noch darauf hingewiesen werden kann, dass Platun (Legg. IX 879 B) die Worte des ersten Frag-

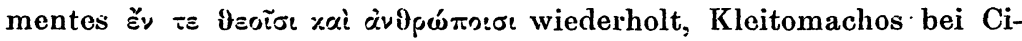
cero (De nat. deor. I 27 f.) auf Fr. 6 sich zu beziehen scheint, in einem Philolaos zugeschriebenen Aussprucbe Fr. 1, 4, 5 benutzt (s. unten S. 343), von Diogenes (IX 19) der zweite Vers des Fr. 1 um-• schrieben wird, so wird man die Echtheit der angegriffenen Verse wohl nicht länger anzweifeln.

Durften wir um der Wichtigkeit des ersten Fragmentes willen eine recht weitschweifige Erörterung nicht scheuen, so wird es degegen gerechfertigt sein, die Untersuchung der minder entscheidenden Bruchstücke in grösserer Kürze zu führen ${ }^{17}$ ).

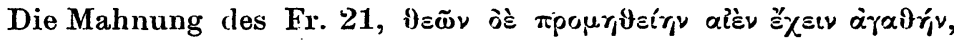
scheint Diels von keinem Belange, weil sie in einer populären Elegie enthalten sei. Aber gerade in diesem, dem grösseren Teile nach sehr ernsten Liede finden wir die Aufforderung, nur Rechtes und Gutes zu sprechen und eine sehr eindringliche Zụückweisung

17) Das Fr. 16, das, wie schon ThdX. (S. 9) erklärt wurde, für die Entscheidung der Streitfrage von geringer Bedeutung ist, übergehe ich. Diels will dasselbe (S. 98) nicht in das Lehrgedicht, sondern in die Sillen des Xenophanes verweisen. Ich habe nie behauptet, class es einen Teil des ersteren gebildet habe, und weiss nicht, welcher,Schrift des Xenophanes es angehört hat. Die Existenz von Sillen des Xemophanes aber hat bisher Niemand erwiesen. 
unwahrer mythologischer Erdichtungen. Wïhrend nun Xenophanes diese als törichtes Geschwätz hinstellt, das auch bei fröhlichem Gelage nicht geduldet werden dürfe, sollte er die viel schlimmere und viel tiefer eingreifende Lehre von der Göttervielheit ruhig hingenommen und selbst empfohlen haben? Das scheint mit der rücksichtslosen Wahrheitsliebe des Dichters schlecht zu stimmen.

Im Fr. 14 erklärt Xenophanes, class niemand in Klarheit kundig sei dessen, was er von den Göttern und vom Weltall sage. Hält man die hergebrachte Auffassung der xenophaneischen Theologie fest, so ist das schlechthin inverständlich. Denn für Xenophanes giebt es keinen Gegensatz von Gottheit und Weltall, und von den Göttern soll er ja in aller Klarheit und Bestimmtheit behauptet haben, dass sie ni cht seien. Erklärt aher Zeller (S. 1596),

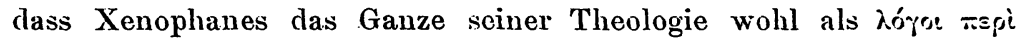

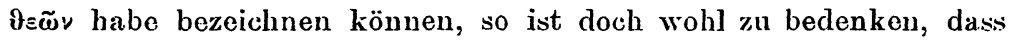
er hier nicht den 'Titel seiner Schrift angiebt, sondern vielmehr behauptet, ,niemand wisse etwas von dem, was er über die Götter sage'. Und' wenn Zeller fortfährt, "class er auch dann, wenn ihm die Einheit und Einzigkeit Gottes feststand, doch die volle Klarheit und Sicherheit' seiner Lchre 'gerade so gut vermissen konnte,

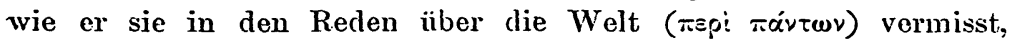
deren Einheit ihm doch gleichtalls zweifellos festistand,' so ist auch hiergegen Manches einzuwenden. Von clen Göttern lehrte er nur, dass sie n i cht seien, und das musste er und mit ihm ein jeder, der seine Lehre annahm, in aller Klarheit und Sicherheit behaupten können. Soll hier aber wiederum der Plural i)sri den Einen Gott bezeichnen, so ist seine Einheit nicht mit ler Einheit der Welt $\% u$ vergleichen. Denn diese Einheit schliesst ja eine unendliche Vielheit der Dinge nicht aus; dort aber soll die Einheit und Einzigkeit der Gottheit mit Ausschluss aller Vielheit gelehrt worden sein. Was konnte bei solcher Einheit ihm unklar bleiben? Auch dies erkennt Diels in rückhaltloser Weise mit den Worten an (S. 98): "Dor Gegensaty von Ver,i und réver ist auf das System des Xenophanes bezogen mir ebenso rätselhaft als der Plural VEo̊'. Aber nicht darum ist das unerklärlich, weil wir, wie Jiels hinzufügt, 'dic Stelle des Gicdichtes nicht keunen, auf die es sich bezicht'. Keines der äbrigen 
floch wohl verstïnllichen Fragmente des xenophaneischen Lehrgedichtes ist uns wach seiner Umgebung und seinern Zusammenhange hekannt, und wo immer das vier\%ehnte Bruchstück gestanden habon mag, immer müissen die Worte der gewöhnlichen Auflasiung als ein unlösbares Problem erscheinen.

Wenn man lie von mir verteidigte Auflassung der xenophatneischen Gotteslehre «u Grunde legt, verschwindet jede Schwierigkeit. Das Woltall in seinem Grunde erfasst, ist Xenophanes sis

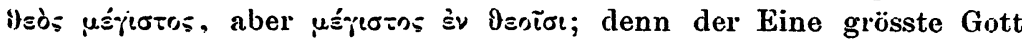
hat neben sich eine Anzahl von Teilgottheiten: das Weltall, identisch mit dem höchsten Gotte, ist also wohl zu unterscheiden von den Osoó. Diese Einheit in der Vielheit aber bot, auch abgesehen ron der Frage nach dem Wesen der Götter, ein Problem dar, das Xenophanes ebenso dunkel erscheinen musste, wie es selbst in den Systemen eines Spinoza, Fichte, Lotze als unlösbares Rätsel auftritt; darum musste Xenophanes freimütig bekennen, dass in Klarheit niemand kundig sei dessen, was er über Götter und Weltall lehre.

Die Fragmente des Xenophanes begründen - das darf nunmehr wohl behauptet werden - die überlieferte Vorstellung von der Gotteslehre des eleatischen Denkers nicht, sondern widerlegen sie. Wie steht es nun aber mit den Angaben späterer Schriftsteller über ihn? Sind sie geeignet, das gewonnene Ergebnis zu erschüttern?

Von geringer Bedeutung ist, wie zugegeben werden muss, Ciceros wohl auf Poseidonios zurïckgehende Angabe (De divin. I 3, 5) Xenophanes unus qui Doos esse diceret ilivinationem funditus sustulit; denn allerdings, Ciceros Zuverlässigkeit ist nicht eben gross, und wird in unserm Falle, wie Zeller hervorhebt, durch die nachfolgende Mitteilung über die Sokratiker noch verringert. Doch denke man auch nicht gar zu gering über den Wert dieses Zeugnisses. Denn war Xenophanes Monotheist, so konnte Ciceros Gewährsmaun ihn nicht wohl behaupten lassen, Deos esse; so würde er ihn eher zu den Atheisten gerechnet haben, wie denn Cicero von Antisthenes ausdrücklich hervorhebt (De nat. deor. I 13, 32) tollit vim et naturam Deorum ${ }^{18}$ ).

18) Diels (S. 99 A.4) hält allerdings Hartfelders Conjectur unum qui Deum, die den Sinn dieser Stelle ins Gegenteil verwandelt, für richtig. Aber diese 
Auch die Worte, die Xenophanes von Aristoteles (Rhet. II 23. 1399b6) in den Mund gelegt werden, sind zwar nicht von entscheidender Bedeutung, aber doch auch nicht zu unterschätzen. Man dürfe, so heisst es hier, weder von Entstehen noch von Vergehen der Götter

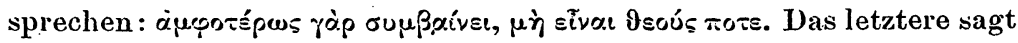
Xenophanes nicht im Sinne seiner Landsleute; denn die Volksreligion nahm ja an dem Begriffe einer Zeit, in der es noch keine Götter gab, nicht den geringsten Anstoss. Er spricht hier im eigenen Sinne, wenn er das 'Nichtsein der Götter in irgend einer Zeit als ungerechtfertigte Consequenz einer irrigen Annahme hinstellt: er selbst kann also die Existenz der $\vartheta$ soi nicht geleugnet haben. Nicht zu vergleichen ist daher die von Aristoteles (das. 1400 b 5) erzählte Anekdote, die den Philosoplien nur im Sinne Fremder sprechen lässt. Und wenn Zeller (S. 1596) auf Aristoteles Eth. Nik. I 10. 1099 b 11; VIII 14. 1162 a 5; IX 1. 1164 b 5 hinweist, als auf Stellen, aus denen man ja auch nicht schliessen dürfe, dass Aristoteles eine Fürsorge der $\vartheta s o \grave{i}$ für die Menschen angenommen habe, so ist zu entgegnen, dass in der nikomachischen Ethik allerdings eine solche mit voller Bestimmtheit gelehrt wird, wie aus $\mathrm{X} 9.1179$ a $24 \mathrm{f}$. hervorgeht. Das widerspricht freilich aufs schärfste anderen unzweideutigen Lehren des Aristoteles und

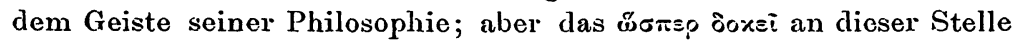
und der Zusammenhang des Ganzen lïsst eine Abschwächung des Gedankens oder eine Auffassung desselben als Ausdruckes der Volks-

kühne Aenderung der überlieferten Lesart ist, soviel ich weiss, von Niemaudem gebilligt worden; sie ist vielmebr dem neuesten Herausgeber Ciceros, C. F. W. Müller so unhaltbar erschienen, dass er sie nicht einmal der Erwähnung für wert gehalten hat. In der That ist sie längst von Schiche (Ztschr. f. Gymnas. Wiss. Jahresber. 1882 S. $23 \mathrm{f}$.) aus grammatischen Gründen zurūckgewiesen worden. Sie ist ferner ohne handschriftliche Grundlage. Unos in B (nicht A nach Deiter Rh. Mus. $1882 \mathrm{~S} .316$; De codic. Voss. p. 36) ist ji identisch mit unus, und Deum in $H$ ist reine Conjectur. Jenn dieser Handschrift, die zahlreiche Vermutungen aufgenommen hat, kommt, wo sie den guten Corlices A B widerspricht, keinerlei Autorität zu, wie aus Vablen (Praef. zu Cicero De legg. " p. VI. XIIf.) und C. F. W. Müller (Jahns Jahrb. 1864 S. 127) hervorgeht. - Bemerkt sei noch, dass ich einer freundlichen Jitteilung des zuletzt genannten Gelehrten die rechte Schätuung der handsi:hriftlichen Celoerliefenung dieser Stelle verdauke. 
vorstellung nicht \%. Fin weiteres Fingehen auf diesen Gegenstand ist freilich hior nieht gestattet.

Wichtiger ist der auf Theophrast zurückgehende Bericht Ps.-

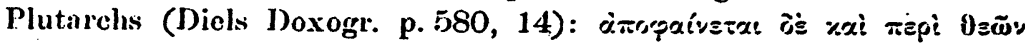

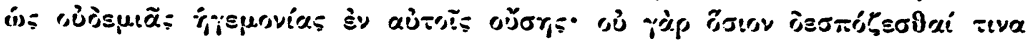

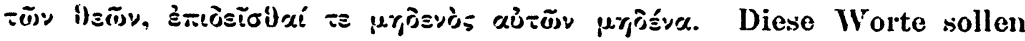
Zeller und Diels zufolge nicht allein kein Bekenntnis des Polytheismus einschliessen, wie es mir erschien, sondern uns auch \%wingen, Xenophanes eine streng monotheistische Anschauung zuzuerkennen. 'Denn (sagt Zeller S. 159 f.) es verhält sich nicht so, wie F. (S. 10) glaubt, dass hier nur der Vorstellung einer despot is ch en Herrschaft des Götterkönigs entgegengetreten würde: sondern

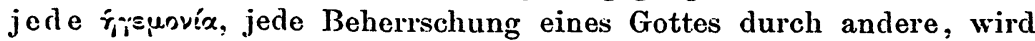
für etwas mit dem Wesen der Gottheit Unvereinbares erklärt: nicht weil ein tyrannisches Regiment ihrer Güte und Vollkommenheit widerstritte, sondern weil einerseits kein Gott einen Herrn

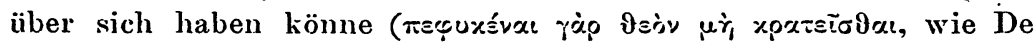
Xenophane 977 a 31 richtig erklärt wird), andererseits Gott als solcher keines IDieners bedürfe'.

Und ähnlich äussert sich Diels (S. 99).

Aber auch diese auf den ersten Blick entscheidenden Beweise dürften schwerlich eingehender Ueberlegung Stich halten. Man gebe einmal $\mathrm{zu}$, dass durch eine strenge Argumentation aus einem Ausspruche des Xenophanes Folgerungen zu ziehen seien, die ihn in einen Widerspruch mit seinen sonstigen Ansichten verwickeln. Sind denn aber derartige Widersprüche bei Philosophen alter und neuer Zeit so selten, dass man um ihretwillen eine sonst gut bezeugte Lehre umdeuten oder gänzlich leugnen dürfte? - Einer der leuchtendsten Vorzüge. der Zellerschen Geschichtswerke besteht darin, dass ihr Verfasser, jeder künstlichen Vertuschung und Verdunkelung unleugbarer Thatsachen abhold, klar und entschieden auf die Inconsequenzen auch der tiefsten Denker hinweist, da wo andere Geschichtschreiber oft genug durch gewaltsame Deutungen eine scheinbare Harmonie zu stiften versucht haben. Schreiend sind die Widersprüche, die Zellers tief begründetes Urteil bei Heraklit und Empedokles, bei Platon und Aristoteles, bei Descartes, Ieibniz, Kant 
und zahlreichen anderen Philosophen ${ }^{19}$ ) nachgewiesen hat. Und Xenophanes, von dessen philosophischen Ansichten nur dürftige Bruchstücke uns Kunde geben, der, mehr Dichter als Philosoph, ein zusammenhängendes System der Philosophie wohl niemals entwickelt hat, dürfte ein. Widerspruch, wie der gerügte, nicht zuzutrauen sein? - Eine ähnliche Inconsequenz, wie sie hier Xenophanes vorgeworfen wird, hat man in spïterer. Zeit auch Chrysippos zum Vorwurf gemacht. Die Gottheit soll ihm zufolge in sich vollkommen und bedürfnislos sein, und mit Recht soll

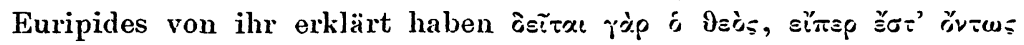
けsòs, ỏ̇òsós. Und doch hat derselbe Chrysipp das Weltall als das einzig Sichselbstgenügende angesehen : die übrigen Götter können also weder sich selbst genügend noch glückselig sein (Plut. De stoic. rep. c. 40). Niemand aber hat um des Mangels an Folgerichtigkeit willen eine dieser Lehren Chrysipp abgesprochen. Warum sollten wir den Dichterphilosophen Xenophanes strenger beurteilen, als den schärfsten Dialektiker der stoischen Schule? - Ein Fragment, das Achilles Tatius gerettet hat und ausdrückliche Angaben Aristoteles' und Theophrasts sagen uns, dass Xenophanes die Luft und den unteren Teil der Erde ins Grenzenlose sich erstrecken liess. Dem widersprechen aber zahlreiche Berichte anderer zuverlüssiger Gew ährsmänner, denen zufolge Xenophanes eine Begrenzung des Weltalls angenommen hatte ${ }^{20}$ ). Haben wir das Recht, um dieses offenbaren Widerspruches willen eine von beiden Angaben zu verdächtigen? Doch wir haben hier ein Zugeständnis gemacht, das viel zu weit geht. In Wirklichkeit besteht kein Widerspruch zwischen den richtig verstandenen Angaben Theophrasts und der von mir vertretenen Ansicht über Xenophanes' Theologie. Freilich, wer annimmt, dass jedes Wort in dem Auszuge Ps.-Plutarchs xenophaneisch sei, der muss den bervorgehobenen Widerspruch anerkennen.

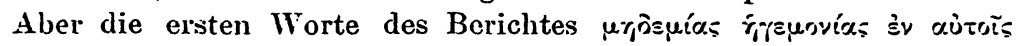

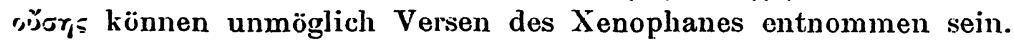

19) S. Philos. d. Griechen I4639. 739. II, $1^{3} 581$. 582. 600f. 704 A. 2 II, $2=196$. 381. 384. 387f. 801. Gesch. der deutschen Philos. S.48. 97. 110. 143 und an anderen Stellen.

(i) Vgl. Zeller Ph. d. Griechen $14.494 \mathrm{f}$. 
Sie klingen fiusserst prosaisch, und dias wichtigste Wort rifeusvix kann aus metrischen firtinden nicht in Versen epischen Masses, in denen Xenophanes' Lehrgedicht abgefasst war, gestanden haben. Lis liegt also eine lediglich dem Berichterstatter angehörige Form iles Gedankens vor. Zu welchen geschichtswidrigen Vorstellungen wir aber gelangen wiirden, wenn wir jeden ungeschickt gewählten Ausclruck, jelle ungenaue Angabe dieser Excerpte als feste Grundlage woiterer Folgerungen ansehen wollten, zeigen die den besprochenen unmittelbar folgenden Worte (Doxogr. 580, 20f.) Пxp-

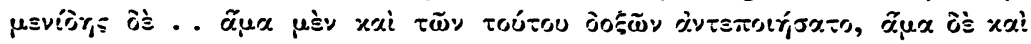

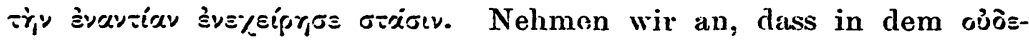

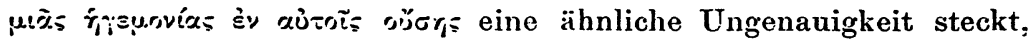
wie in diesem über Parnenides abgegebenen und vielen anderen Urteilen; dass jene Worte nui eine ungenaue Ümschreibung des

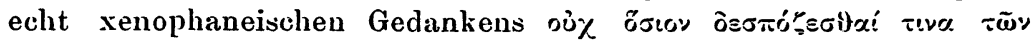

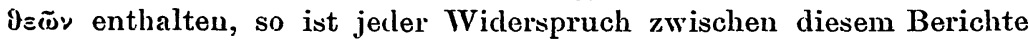
und der bestrittenen Lehre ron der Göttervielheit verschwunden. Daun hat Senophanes nicht jede Unterordnung der Teilgötter unter den Einen höchsten Gott, sondern nur die despotische Beherrschung der unteren Götter bekämpfen wollen; ja vielleicht hat er hier, wie in der angeschlossenen Erklärung über die Bedürfnislosigkeit der Götter, nur die sehr anstössigen Erzählungen griechischer Dichter von der despotischen Regierung des Götterkönigs zurückgewiesen ${ }^{2}$ ). Dass diese Erklärung der Ansicht des Xenophanes entspricht, beweist Euripides, der in offenbarer Nachbildung des xenophaneischen Gedankens, die Worte spricht (Her. fur. 1343)

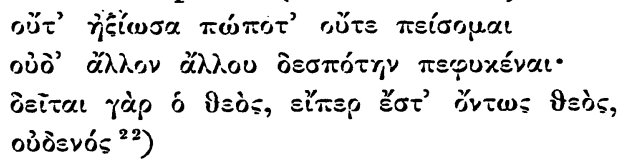

21) Die im 'lexte angegebene Bedeutung von $\delta \varepsilon \sigma \pi \delta_{\sigma}^{\prime} \varepsilon \sigma q \alpha \iota$ ist bekannt ge-

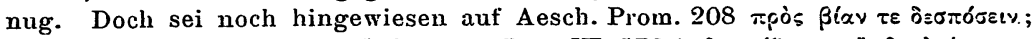

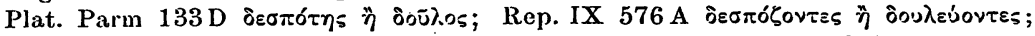

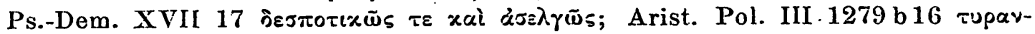

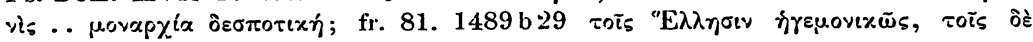

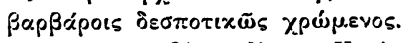

:2) Der Sinn dieser Verse ist klar. Theseus hatte Ilerakles, un ihn von 
Sowenig wir nun Euripides den Glauben an die Existenz einer Göttervielheit und eines höchsten Gottes absprechen, weil er keinen Gott unter die despotische Herrschaft eines andern stellen will ${ }^{23}$ ), so wenig sind wir gezwungen, Xenophanes' Lehre von Einem höchsten und vielen Nebengottheiten für unvereinbar mit diesem Gedanken zu erklären. Denn Xenophanes lehrt hier nichts anderes, als was ein Neupythagoreer in den Worten bei Stobaeos

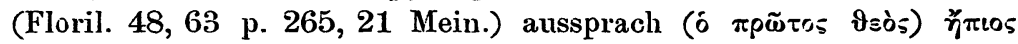

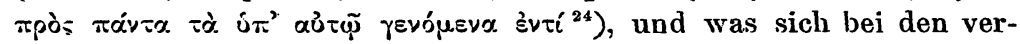
schiedensten anderen Schriftstellern findet.

Wenn die bisher untersuchten Fragmente und Berichte mit den Ergebnissen meiner Abhandlung in vollem Einklange stehen, so fragt sich nur noch, ob auch die bekannten Angaben Aristoteles' (Metaph. A5. 986 b24) und Theophrasts (Doxogr. 480, 4) mit den-

seinem Entschlusse, Hand an sich selbst zu legen, abzulenken, auf das Beispiel der Götter hingewiesen, die trotz aller gegen einander begangenen Frevel den Olymp bewohnen und ihre Schuld leicht $z u$ tragen verstehen. V. 1301 :

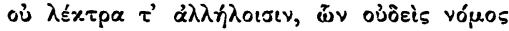

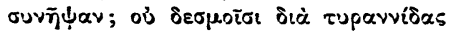

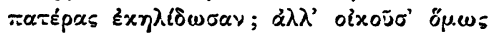

$$
\begin{aligned}
& \text { "Oגopisov } x \tau \lambda \text {. }
\end{aligned}
$$

Darauf entgegnet Herakles (V. 1328f.)

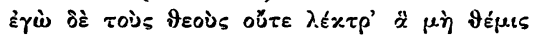

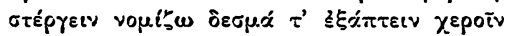

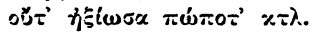

Die Wahrheit jener Erzählungen von den Ehebrüchen der Götter, von der Fesselung der Väter durch ihre Söhne bestreitet also Herakles, und er verallgemeinert das òt⿳亠 zupavvís $\alpha$ s (in V. 1304) durch die Erklärung: 'Kein Gott übt Despotenherrschaft über den andern'. Das Ganze dieser Entgegnung wird sodann durch den Satz begründet: 'Denn die Gottheit, wenn sie in Wahrbeit Gottheit ist, ist bedürfnislos'. Die Existenz der Götter aber soll so wenig geleugnet werden, wie die hohe Stellung des Zeus im Kreise der Götter. Beides ist vielmehr die notwendige Voraussetzung des Dramas. Dass Euripides sie in diesen Versen zu Gunsten einer philosophisch-monotheistischen Theorie aufgeholen habe, wird wohl Niemand behaupten.

23) Ueber Euripides Stellung zum Volksglauben s. Zeller Ph. d. Gr. II ${ }^{3} 15$ f.

24) Jan könnte hier auch die dem Polytheisten Ekphantos zugeschriebe-

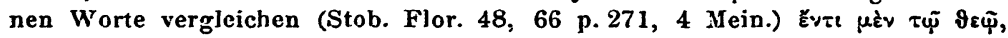

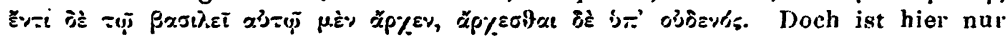
vom hïchsten Gotte die Rede.

Archiv f. Genchichte a. Philosoplie. I. 
selben zu voreinigen sind. Zeller bestreitet dies. 'Aristoteles und 'Thoophrast', so crklärt er (S. 1597), 'legen Xenophanes die Lehre von der Einzigkeit Cottes mit einer Bestimmtheit bei, der sich nichts abdingen lïsst. Wenn ler Erstere von ihm sagt: to डิ siva

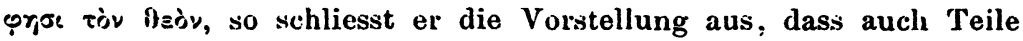
dieses Einen Götter sein könnten; denn wenn die Gottheit das Eine ist, kann sie nicht zugleich eine Mehrheit sein. Wenr Theophrast

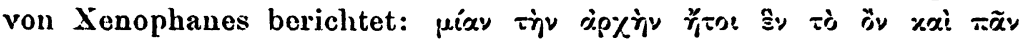

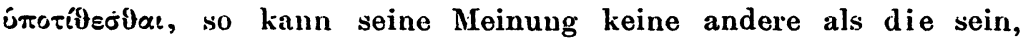
dass die ¿̇pxì nur Eine sei, wie sie dies ja auch sein muss, wenn Gott (wie Theophrast sofort beifügt) das ầ xxi $\pi \tilde{a}$ ist'.

Beweisen diese Stellen aber wirklich, was sie beweisen

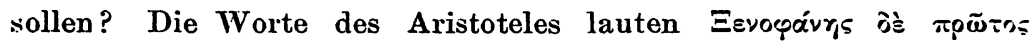

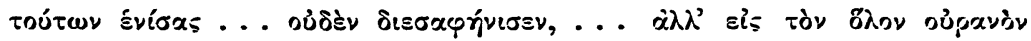

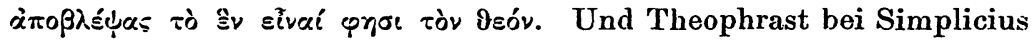

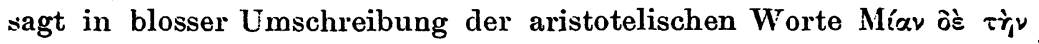

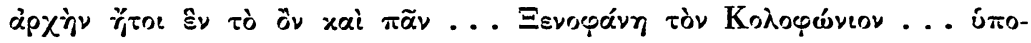

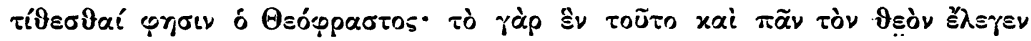
ó Esvọ̣ávis. Xenophanes hat also das Weltall als Einheit erfasst

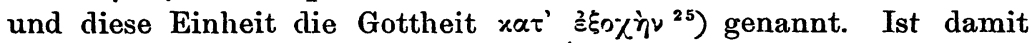
die Vielheit der Götter geleugnet? So wenig, sollte man glauben, als die Einheit der Welt die Vielheit der Einzeldinge nach Xenophanes ausschliesst. Denn nur von der Einheit des metaphysischen Principes handelt Aristoteles in diesem Abschnitte seiner Metaphysik; nur von ihm kann Theophrast sprechen. Wie wohl verträglich aber diese Einheit mit der Vielheit göttlicher und ungöttlicher Individuàlitäten ist, geht aus dem Umstande klar hervor, dass Aristoteles das §ิ «นi $\pi \tilde{x \nu}$ selbst bei den ältesten ionischen Philosophen findet (Metaph. A 8. 988 b 22; 3. 984a27f.; vgl. Bonitz z. St.). Für die Richtigkeit dieser Deutung weise ich nicht auf das von mir (ThdX. S. $20 \mathrm{f}$.) Ausgeführte hin; ich rufe das Zeugnis

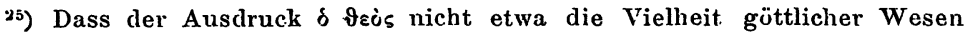
ausschliesst, lehrt am eindringlichsten Lehrs Popul. Aufsätze $2147 \mathrm{f}$. - Ein-

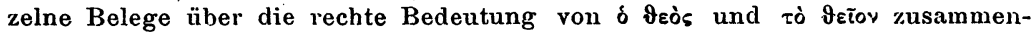
zutragen, erscheint, da fast jedes Blatt der griechischen Litteratur sie darbietet, überflüssig. 
Zellers selbst an, der die entscheidenden Worte spricht (Ph. d. Gr. III $2^{3} 115$ f.): 'Die Einheit der höchsten Ursache ist allgemeine selbstverständliche Voraussetzung; doch hindert dies unsere Pythagoreer so wenig, als andere, zugleich auch von den Göttern als einer Mehrzahl zu spreçen, und der strengere Monotheismus, welcher sich weigert, neben dem höchsten Gott weitere Götterwesen anzuerkennen, wird sogar ausdrücklich zurückgewiesen, indem neben dem Einen unsichtbaren Gott in den Gestirnen sichtbare Götter anerkannt werden, die in seinem Dienst stehen ${ }^{26}$ ). - Aehnlich urteilt Zeller über ein Philolaos zugeschriebenes Fragment bei Philon (De m. opif. p. 24, $10 \mathrm{f}$. M.) غ̇ऽì ràp

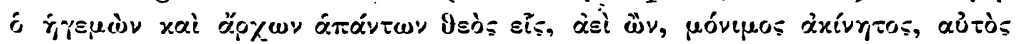

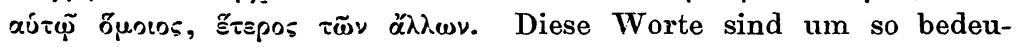
tungsvoller, je näher sie den xenophaneischen Bruchstücken stehen.

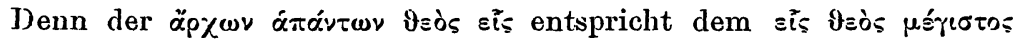
des Xenophanes; die Worte róvequs d'xívitos dem Fr. 2 aisi $\hat{o}^{\prime}$ हैy

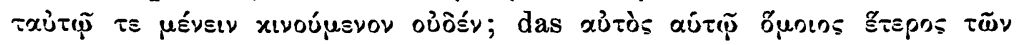

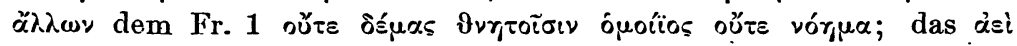
¿̈y endlich dem V. 1 des Fr. 5 und dem ähnlichen Ausspruche bei Aristoteles (oben S. 337). Aber nicht die Lehre des Monotheismus findet Zeller in diesem Bruchstücke ausgesprochen. 'Würde endlich', so ïussert er sich ( $\mathrm{Ph}$. d. Gr. $\mathrm{I}^{4} 345,1$ ) ' ein strenger Monotheismus allerdings dem theologischen Standpunkt der Pythagoreer widersprechen, so fragt es sich doch, ob unser Bruchstück in die-

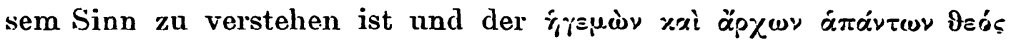
andere Götter ausschliessen soll, ob wir daher hier mehr haben, als jenen mit' dem Polytheismus nicht unverträglichen Glauben an einen böchsten allwaltenden Gott, wie wir ihn auch vor und neben Philolaus bei einem Aeschylus, Sophokles, Heraklit, Empedokles und anderen finden'.

Wenn der Mann, der tiefer als irgend Einer der jetzt Lebenden in den Sinn der griechischen Gedankenwelt eingedrungen ist, dies Urteil abgiebt, dann dürfte eine weitere Begründung meiner Erklärung der aristotelischen Angaben wohl überflüssig erscheinen.

"9 Vgl. auch Zeller Ph. d. Gr. $1^{4} 738$. II $3^{3} 146$. III $1^{3} 315$. 318. $1112^{3} 126$ u. 138.207 f. 226 u. 229.688 f. 792 f. u. s. 
Doch sei Einzelnes aus einer unübersehbaren Zahl von Belegen als Beweis fïr die Thatsache hervorgehoben, dass griechische Denker rer verschiedensten Zeiten mit der Ueber\%eugung von der Einheit eines höchston güttlichen P'rincipes die Anerkennung und Verehrung der Einzelgötter zu vereinigen gewusst haben.

Ein pseudo-orphisches Gedicht, dessen Grundbestandteile sehr alt sind und dessen spätere Zusïtze durchaus im Geiste der alten Dichtungen gehalten sind, schildert die Alleinheit des Zeus (p. 202

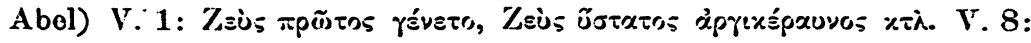

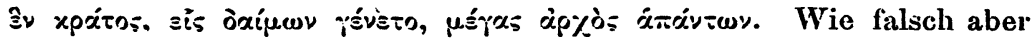
wïre der Schluss, den man aus diesen Versen zu ziehen alle Teranlassung hätte, dass dem Dichter alle Vielheit der Götter in der Einheit des Allumfassers Zeus verschwunden sei. Denn von $t=\tilde{\omega}$, óò sủpxvé́vwr spricht V. 17; und während Alles in Zeus enthalten ist, sollen doch die Einzelwesen endlicher und göttlicher Art ihre Sonderexistenz behalten (V.9)

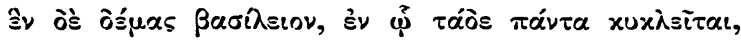

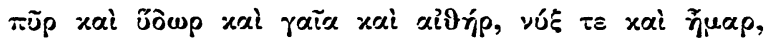

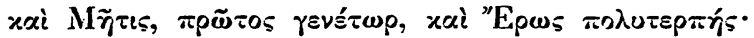

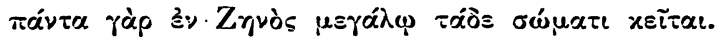

Aristoteles selbst spricht die Einzigkeit seines göttlichen Princips aufs entschiedenste aus, und doch hat er eine Vielheit gött-

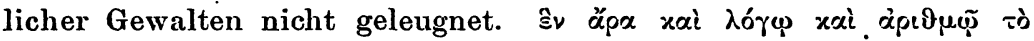

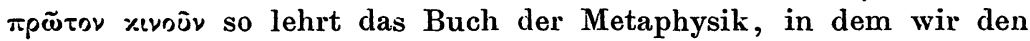
Abschluss der gesammten aristotelischen Philosophie erblicken müssen ( $\Lambda$ 8. 1074a36); sís xoípavos, so schliesst dies Buch die Erörterungen über die höchste Gottheit. Aber daneben erkennt Aristoteles die göttliche Natur der Gestirne und damit die Vielheit der Götter ausdrücklich an; ja in einem einzigen Satze, der unsrer Anschauung nach Unvereinbares vereinigt, spricht er aus, was ihm zugleich als Grundgedanke griechischer Religion erschien und als

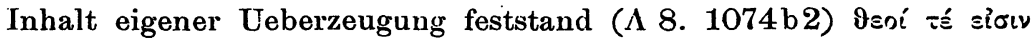

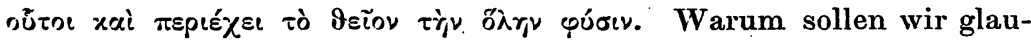
ben, dass Xenophanes die Consequenz seiner Lehre vom ७sìs uś-

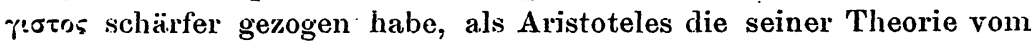

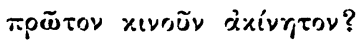


In einem einzigen göttlichen Principe erblicken die Stoiker Ursache, Substanz und Vernunft der Welt; sie erklären die Welt für eine Einheit, für identisch mit der Gottheit, die selbst nur Eines sein könne. Eine Reihe von Citaten, wie wir sie bei Alexander (De mixt. 142 a), Diogenes (VII 148), Sextus (Adv. Math. IX 79), Eusebios (Pr. ev. XV 15) u. A. ${ }^{27}$ ) finden, zwingen uns ganz dasselbe als Lehre der Stoiker anzusehen, was Aristotele

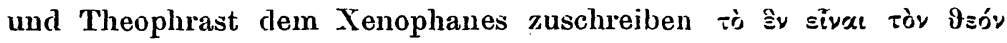

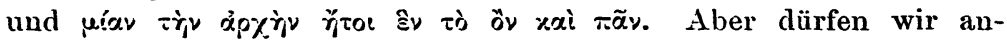
nehmen, dass, weil ihnen die Gottheit das Eine ist, eine Mehrheit von Göttern geleugnet wird? Nein! Aus dem Einen ewigen Urgrunde gehen andere göttliche Individualitïten hervor, mit deren Annahme das Vielgöttertum der Volksreligion - bei aller Abweisung seiner denkwidrigen Auswïchse - sich wohl vereinigen liess.

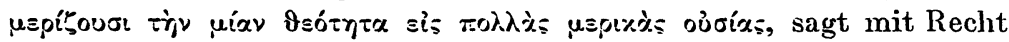
Epiphanios (Adv. haer. I 5) von den Stoikern, und Plutarch be-

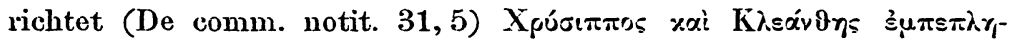

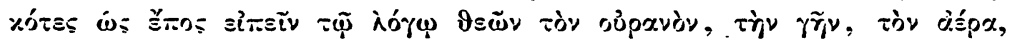

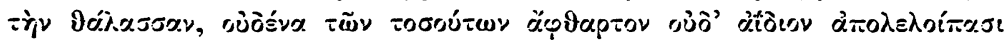

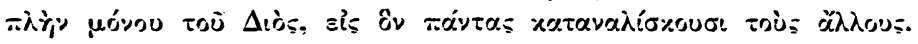

Den Stoikern sich anschliessend erklärt Marc Aurel (Ne-

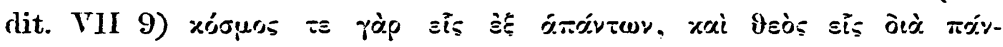

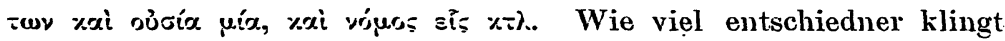
das im Sinne eines strengen Monotheismus als der aristotelische Bericht über Xenophanes' Gottheit. Aber zahllos sind die Stellen, in denen Marc Aurel seine Ueberzeugung von der Existenz der Einzelgötter ausspricht. Und in der That, nie wiirde der edelste der Cäsaren ohne liese Ujeberzeugung lediglich aus politischen Motiven jene furchtbare Verfolgung über die Bekenner des Monotheismus verhängt haben, die sein Andenken befleckt.

In ähnlichen Ausdrücken wie Marcus Aurelius hat Plutarch seinen Glauben an ein allumfasseudes göttliches Princip ausge-

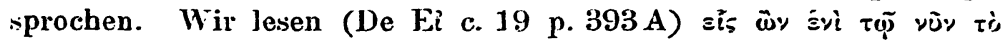

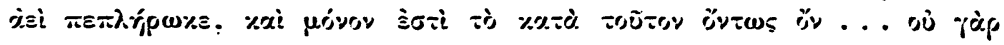

\#) Vgl. Zeller IIl $1^{3} 119.137$ f. 167 f. 310 f. 315 u. s, 


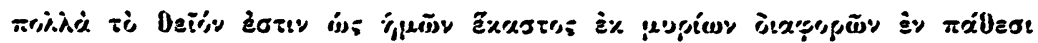

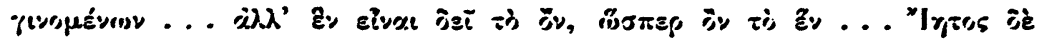

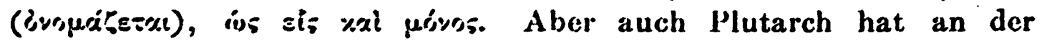
Vielhoit der Götter nicht gezweifelt, wie wiederum Zeller hervorhobt. (Ph. d. Gr. III $2^{3}$ 190f.).

Mit grösster Entschiedenheit hat endlich Plotin die Einheit und Einzigkeit der Gottheit betont, äber jegliche Vielheit und Zusammenset\%ung, ïber Gleichheit und Achnlichkeit mit irgend einem anderon Wesen sic orhoben. Aber derselbe Plotin ist es, der

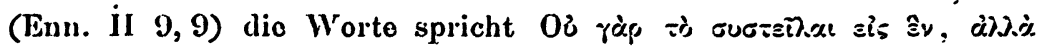

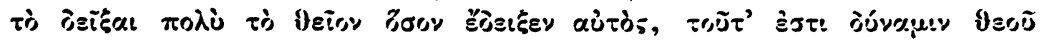

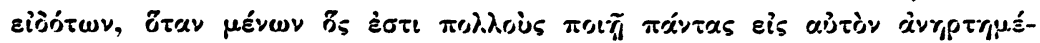
veus \%थג.

Die Menge dieser Belege muss die Ungeduld des kundigen Lesors erregen; aber sie beweist uns in unwiderleglicher Weise, in wic vielfachen Modificationen der griechische Polytheismus mit dem Glauben an die Finheit des göttlichen Principes sich verbunden hat. Sie giebt uns zugleich Antwort auf Zellers Frage (S. 1597), 'wo der Kolophonior, die ihm zugeschriebenen ewigen Wesenheiten in seiner Wolt unterbringen und was ihn zu ihrer Annahme veranlassen' konntc. Sic anzunehmen veranlasste Xenophanes dersolbe Grund, der auch andore tiefore Geister noch in späterer Zeit, da der monotheistische Geclanke viel mehr betont ward, an der Vielheit dor Götterwesen festhalten liess: der Zwang, dem selbst die freiesten und. kühnsten Denker unterliegen, das allgemeine Bcwusstsein des Volkes, dem sie angehören, dessen Anschauungen selbst dann noch in ihnen fortwirken, wenn sie sich denselben zu entwinden suchen. - Wo der Kolophonier seine Einzelgötter unterbrachte, das genau zu bestimmen, sind wir ausser Stande; denn so vielfach die erwähnten Ansichten über das Wesen der Götter sind, die neben das allumfassencle göttliche Princip gestellt werden, so viele und vielleicht noch mohr Möglichkeiten giebt es, den Göttern des Xenophanes ihren Platz in seiner Philosophie anzuweison. Wenn einigo dieser Möglichkeiten von mir herausgegriffen worden sind und ihre Vereinbarkeit mit den iibrigen Lehren des Xenophanes nachzuweisen versucht wurde (ThdX. S. 30), so habe 
ich doch die Unsicherheit eines solchen Versuches offen anerkannt.

Aber mögen auch die Einzelheiten der xenophaneischen Gotteslehre dunkel sein und bei der Dürftigkeit der geschichtlichen Ueberlieferung wohl immer bleiben, klar scheint zu sein, dass der Begründer der eleatischen Schule eine streng monotheistische Lehrc nicht ausgesprochen hat. Auch im Gegensatze zu Zeller und Diels scheint festgehalten werden zu müssen, dass Xenophanes aus der Reihe der griechischen Denker nicht ganz herausgetreten ist, dass er die Anschauungen seines Volkes von der Gottheit veredelt, aber nicht gänzlich aufgegeben hat. 